\title{
Reconstruction cutanée et communications tissulaires
}

La reconstitution in vitro de la peau humaine est maintenant possible par implantation d'un explant épidermique sur un équivalent du derme constitué d'un gel de collagène de type 1 remanié par des fibroblastes. Outre son considérable intérêt thérapeutique pour le traitement des malformations cutanées étendues et des brûlures graves, cette technique constitue un formidable outil d'analyse physiopathologique permettant d'analyser la contribution respective des cellules du derme et de l'épiderme dans l'organisation et la différenciation normales et pathologiques de la peau. Il est possible, grâce au modèle de reconstitution in vitro présenté ici, d'aborder aussi le problème de la coopération cellulaire et de son intervention dans la réponse tissulaire au cours de la cicatrisation ou de l'hypersensibilité.

\section{Bernard Coulomb \\ Chargé de recherche à l'Inserm}

\section{Louis Dubertret}

Professeur à la Faculté

Directeur de l'unité U.312 de l'Inserm

\section{ADRESSE}

B. Coulomb, L. Dubertret: laboratoire de dermatologie, Inserm U. 312, hôpital HenriMondor, 94010 Créteil, France.

\section{TIRÉS A PART}

L. Dubertret: laboratoire de dermatologie, Inserm U. 312, hôpital Henri-Mondor, 94010 Créteil, France.

$\mathrm{m} / \mathrm{s} n^{\circ} 2$ vol. 4, feurier 88 'importance des communications cellulaires en physiologie et en pathologie est de plus en plus évidente. Il est clair également que les cellules s'expriment différemment selon leur environnement. Il semble donc nécessaire de disposer de modèles permettant d'aborder les problèmes de communication entre des cellules placées dans leur environnement physiologique, c'est-à-dire d'étudier les problèmes de communications entre tissus. Ceci est d'autant plus important que ces problèmes de communications tissulaires sont au cœur de la compréhension de l'organogenèse pendant la vie embryonnaire et sont responsables de la sauvegarde des structures tissulaires pendant la vie adulte.

La peau est un organe privilégié pour aborder ces questions. En effet, cet organe étendu, accessible à l'observation et aux prélèvements, est constitué de deux tissus d'origine embryologique et de fonction très différentes : le derme et l'épiderme.

Le derme, d'origine mésenchyma- teuse, est constitué essentiellement de fibroblastes qui synthétisent et dégradent une abondante matrice extracellulaire faite de collagène, d'élastine, de protéoglycanes et d'acide hyaluronique. Le derme est traversé par des capillaires et des terminaisons nerveuses qui assurent sa nutrition et son innervation ainsi que celle de l'épiderme sus-jacent. On comprend donc que le derme ait été longtemps considéré comme un simple tissu de support assurant les besoins métaboliques de l'épiderme sus-jacent.

L'épiderme, d'origine ectodermique, est constitué d'une phase cellulaire continue, contenant $95 \%$ de kératinocytes qui se renouvellent en permanence à partir d'une couche basale germinative située au contact du derme. Puis les kératinocytes, ayant fini de se diviser, migrent vers la surface cutanée en subissant des processus de maturation de plus en plus rapides les transformant en cellules plates anucléées, les cornéocytes. Ces cornéocytes sont fortement attachés les uns aux autres et forment une barrière imperméable essentielle à notre survie, 


\section{RÉFÉRENCES}

1. Sengel P. Morphogenesis of skin. Londres : Cambridge Univ Press, 1976 : 1-269.

2. Cohen J. Transplantation of hair papillae. Symp Zool Soc Lond 1966; 12 : 331.

3. Jahoda C, Horne KA, Oliver R F. Induction of hair growth by implantation of cultured dermal papilla cells. Nature 1984 ; 311 : 560-2.

4. MacKenzie I, Fusenig NE. Regeneration of organized epithelial structure. J Invest Dermatol 1983 ; 81 : 189s-94s.

5. Briggaman RA. Epidermal-dermal interactions in adult skin. J Invest Dermatol 1982 ; $79: 21 s$.

6. Bell E, Ivarsson B, Merrill C. Production of a tissue like structure by contraction of collagen lattices by human fibroblasts of different proliferative potential in vitro. Proc Natl Acad Sci USA 1979; 76 : 1274-8.

7. Sarber R, Hull B, Merrill C, Soranno T, Bell E. Regulation of proliferation of fibroblasts of low and high population doubling levels grown in collagen lattices. Mech Ageing Dev 1981 ; 17 : 107-17.

8. Coulomb B, Dubertret L, Bell E, et al. Endogenous peroxidases in normal human dermis : a marker of fibroblast differentiation. J Invest Dermatol 1983 ; 81 : 75-8.

9. Nusgens B, Merrill C, Lapière C, Bell E. Collagen biosynthesis by cells in a tissue equivalent matrix in vitro. Coll Relat Res $1984 ; 4: 351-64$.

10. Coulomb B, Dubertret L, Bell E, Touraine $R$. The contractility of fibroblasts in a collagen lattice is reduced by corticosteroids. la couche cornée. Ce processus de maturation est fréquemment comparé à celui de la lignée érythrocytaire.

L'épiderme comporte également des cellules provenant de la crête neurale, les mélanocytes, responsables de la pigmentation. Il comprend aussi des macrophages provenant de la moelle osseuse, les cellules de Langerhans qui sont les plus puissantes cellules présentatrices d'antigènes de l'organisme. L'épiderme est également à l'origine de formations annexielles, véritables bourgeons épidermiques, pénétrant dans le derme: ce sont les glandes sudorales, les glandes sébacées et les poils.

C'est Ph. Sengel à Grenoble [1] qui, le premier, a montré que, durant la vie embryonnaire, la formation des annexes épidermiques était sous le contrôle d'influences dermiques. Des influences dermiques variées, dépendantes de la localisation anatomique et de l'âge de l'embryon, orientent l'épiderme vers tel ou tel type de différenciation. Ces influences existent également chez l'animal adulte et c'est le mérite de J. Cohen [2] d'avoir, à travers des travaux remarquables sur les papilles pilaires, démontré le rôle du derme dans l'induction de la formation de poils. Ses travaux ont été développés et confirmés par l'équipe de R.F. Oliver et en particulier par C. Jahoda [3] qui ont mis en évidence le rôle privilégié des fibroblastes de la papille folliculaire dans l'induction des événements épidermiques aboutissant à la formation d'un poil.

Enfin, grâce à une méthode de culture permettant de mettre en communication des cellules épidermiques et différents éléments dermiques par l'intermédiaire d'une membrane de collagène, I. MacKenzie et N.E. Fusenig [4] ont prouvé l'importance des facteurs dermiques, non seulement dans la croissance, mais également dans la différenciation des kératinocytes.

Ces travaux de physiologie ont été étendus à des études physiopatho- logiques tendant à mieux comprendre l'origine dermique ou épidermique des maladies héréditaires cutanées. Ces expériences procèdent en général en deux étapes : tout d'abord greffe de la peau malade sur la souris nude* et vérification de la persistance de la maladie génétique après greffe, ce qui prouve que l'anomalie génétique est bien située dans la peau et non pas due à l'expression au niveau de la peau d'une anomalie systémique. Puis, dans un second temps, des recombinaisons variées entre derme et épiderme normal et pathologique permettent de mieux comprendre l'origine dermique ou épidermique de la maladie. Ainsi, il a pu être montré que l'ichtyose lamellaire (ichtyose congénitale sévère) est d'origine épidermique [5].

Il devenait ainsi de plus en plus clair que la construction et le maintien des structures de la peau dépendaient, entre autres facteurs, d'un dialogue constant entre le derme et l'épiderme. Reste que le derme est une structure complexe et qu'un système de culture permettant une étude analytique des interactions cellulaires dans des conditions physiologiques devenait donc nécessaire pour pousser plus loin les investigations. C'est dire l'intérêt soulevé par la mise au point d'un ensemble de techniques permettant de reconstruire in vitro un équivalent de la peau humaine.

\section{Reconstruction de la peau humaine in vitro}

Tout a commencé en 1978 dans le laboratoire d'E. Bell au MIT (Massachusetts institute of technology) à Boston [6]. Un étudiant, cultivant des fibroblastes dans une matrice de collagène, a eu la surprise de voir les fibroblastes interagir avec les fibres de collagène, contracter progressivement le gel initial formé par le mélange fibroblastes-collagène, pour former progressivement un disque résistant au sein duquel les fibro-

\footnotetext{
* Souris nude: souris athymique, immunodéprimée, tolérante par conséquent aux allo-et hétérogreffes.
} 


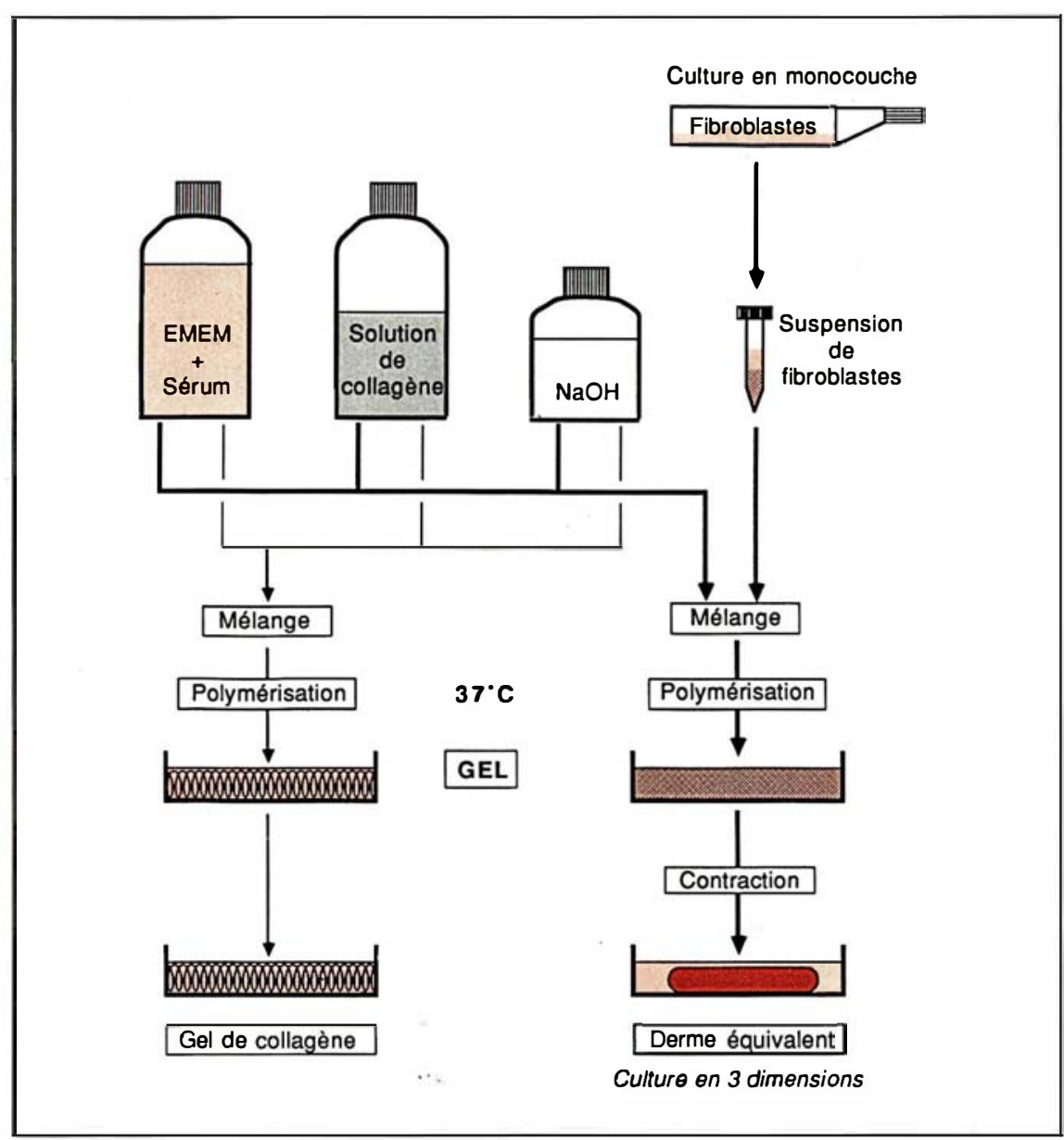

Figure 1. Reconstitution d'un derme équivalent. Des fibroblastes, mis en culture à partir de dermes humains, sont multipliés en utilisant la technique classique de culture en monocouche. Les cellules adhèrent donc au plastique de culture et se divisent jusqu'à ce que toute la surface du flacon de culture soit couverte. Lorsque cette confluence est atteinte, les cellules sont alors en arrêt de division, et pour continuer à en augmenter le nombre, il est nécessaire de détacher les fibroblastes par une enzyme, la trypsine, et de les réensemencer sur un support plus grand où ils vont pouvoir reprendre leur multiplication. Pour réaliser un derme équivalent, des fibroblastes provenant de cultures en monocouche sont mis en suspension dans du milieu de culture et associés à du collagène de type I en solution, dont on neutralise l'acidité, ainsi qu'à un milieu nutritif. II se forme alors un gel dans lequel les fibroblastes sont répartis uniformément. En l'absence de cellules, le gel de collagène polymérise, mais l'on observe aucune modification dans la taille de ce gel. En revanche, les fibroblastes présents dans la matrice de collagène interagissent avec elle. Ils la réorganisent, la contractent et en expulsent le milieu de culture, ce qui aboutit à la formation d'un tissu manipulable, le derme équivalent. Dans ce système de culture en trois dimensions, les fibroblastes retrouvent une différenciation très proche de celle qu'ils expriment in vivo. (EMEM = milieu minimum de Eagle avec sels de Earle)

blastes développaient de longs prolongements cellulaires, s'accrochant aux fibres de collagène, exactement comme ils le font in vivo dans la peau humaine.

Le derme. Cette découverte a été à l'origine d'une série de travaux destinés à savoir si les fibroblastes cultivés dans ces conditions se $\mathrm{m} / \mathrm{s} n^{\circ} 2$ vol. 4, féurier 88 inclus dans leur matrice, ne se divisent plus que pendant 5 ou 6 jours, puis une inhibition des mitoses se manifeste alors que les cellules restent bien séparées les unes des autres tout au long de la culture [7]. Ce modèle du derme équivalent permet donc d'explorer les messages contrôlant les divisions à l'intérieur d'un tissu. Nous avons montré que la perméabilité membranaire des fibroblastes du «derme-équivalent» était semblable vis-à-vis de certains réactifs à la perméabilité membranaire des fibroblastes du derme humain vivant et très différente de la perméabilité membranaire des mêmes fibroblastes cultivés en monocouche[8]. Nous avons montré également que des enzymes de différenciation, impliquées dans la synthèse des prostaglandines, s'exprimaient dans les fibroblastes du derme équivalent ainsi que dans les fibroblastes du derme humain normal et ne s'exprimaient pas dans les mêmes fibroblastes cultivés en monocouche [8]. Enfin l'équipe de $\mathrm{Ch}$. Lapière et B. Nusgens à Liège [9] a montré que les fibroblastes dans le derme équivalent étaient capables de poursuivre la synthèse du collagène de façon beaucoup plus complète que ces mêmes fibroblastes cultivés en monocouche. Ainsi, avec le derme équivalent, nous disposons de fibroblastes différenciés que $\mathrm{Ch}$. Lapière appelle volontiers des fibrocytes pour souligner leur étroite parenté avec les fibroblastes différenciés du derme humain normal. Ce derme équivalent est actuellement très utilisé pour des études physiologiques ou physiopathologiques. En effet, ce modèle permet d'étudier la contraction des fibroblastes [10], fonction cellulaire qui n'était pas accessible à l'expérience jusqu'à présent et permet également d'étudier les interactions cellules/matrice. Il permet enfin d'étudier le comportement de fibroblastes génétiquement anormaux, comme ceux de certaines épidermolyses bulleuses [11] dans lesquelles une 


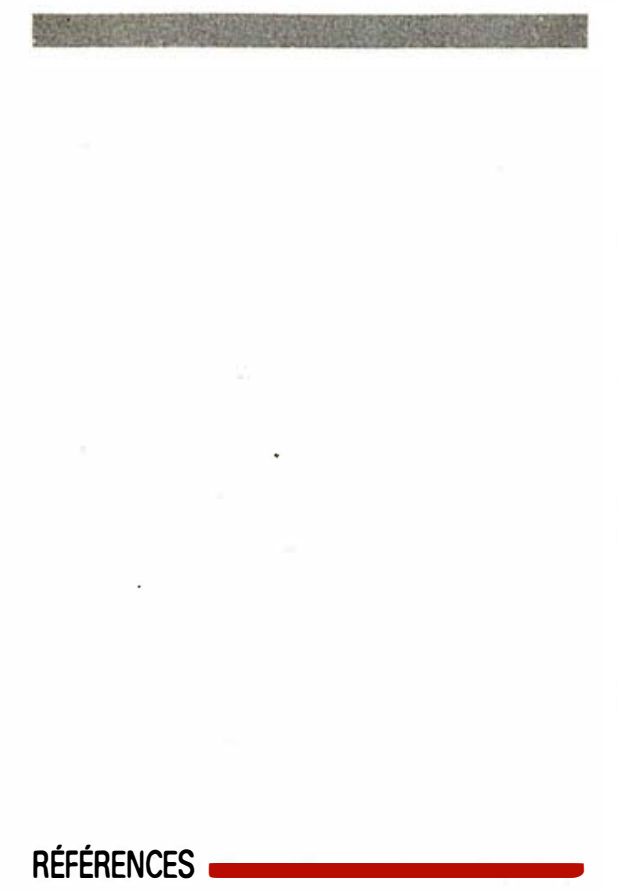

11. Ehrlich HP, Buttle DJ, Trelstad RL, Hayashi K. Epidermolyse bullosa dystrophica recessive fibroblasts altered behaviour within a collagen matrix. J Invest Dermatol 1983; $80: 56-60$.

12. Bauer E, Gedde-Dahl T, Eisen AZ. The role of human skin collagenase in epidermolysis bullosa. J Invest Dermatol 1977 ; 68 : 119 24.

13. Rheinwald JG, Green H. Serial cultivation of strains of human epidermal keratinocytes: the formation of keratinizing colonies from single cells. Cell $1975 ; 6$ : 331-44.

14. Bell E, Ehrlich P, Sher S, et al. Development and use of a living skin equivalent. Plast Reconstr Surg 1981 ; 67 : 386-92.

15. Coulomb B, Saiag P, Bell E, et al. A new method for studying epidermalization in vitro. Br J Dermatol 1986; 114 : 91-101.

16. Saiag P, Coulomb B, Lebreton C, Bell E, Dubertret L. Psoriatic fibroblasts induce hyperproliferation of normal keratinocytes in a skin equivalent model in vitro. Science $1985 ; 230: 669-72$.

17. Fraki JE, Briggaman RA, Lazarus GS Transplantation of psoriatic skin onto nude mice. J Invest Dermatol 1983 ; 80 : 31 s-5s.

18. Bell E, Moore H, Mitchie C, Sher S Coon $\mathrm{H}$. Reconstruction of a thyroid gland equivalent from cells and matrix materials. Exp Zool 1984 ; 232 : 277-85.

19. Weinberg CB, Bell E. A blood vessel model constructed from collagen and cultu-

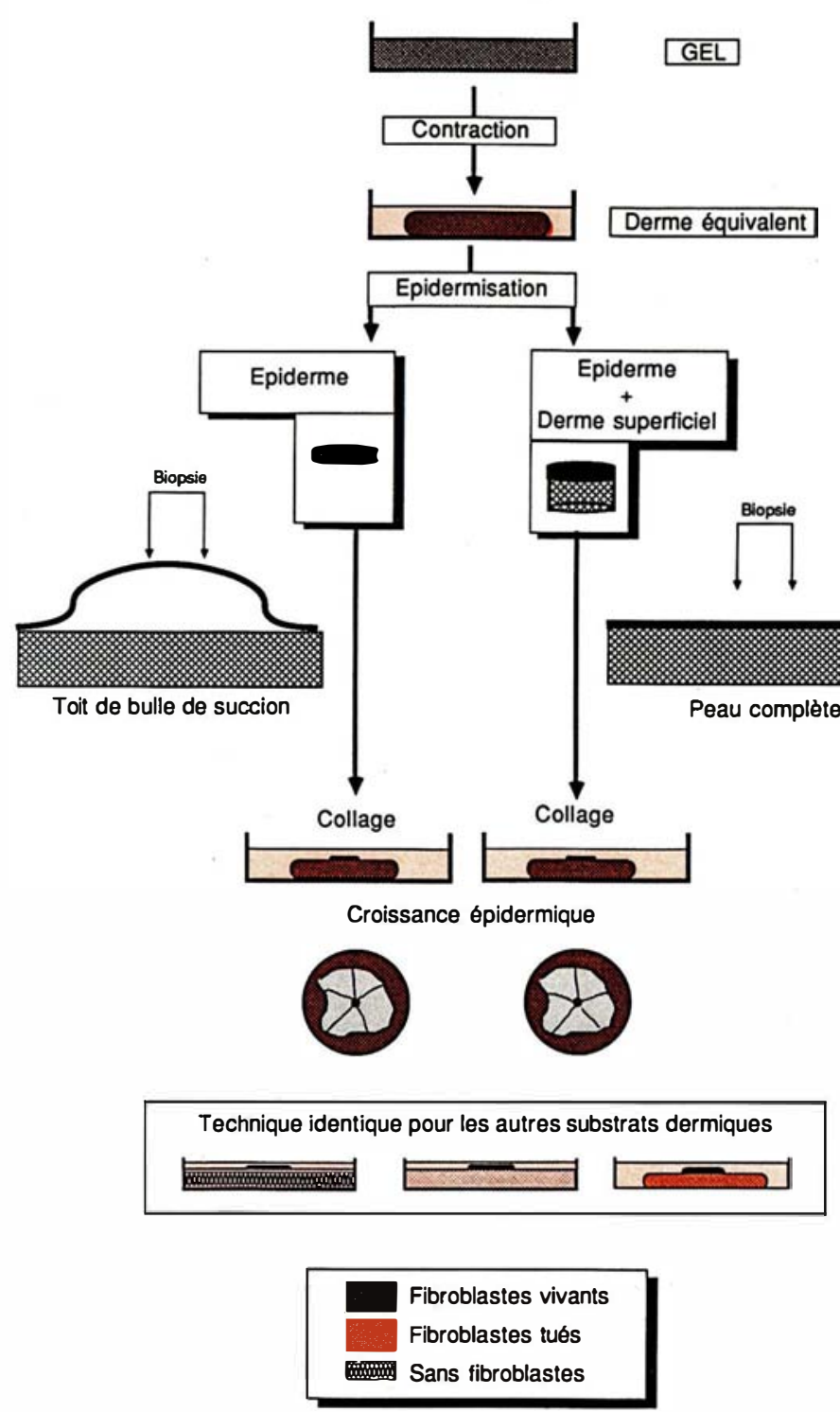

Figure 2. Reconstitution d'une peau équivalente. La réalisation d'une peau totale doit parvenir, tout comme in vivo, a l'association d'un derme et d'un épiderme. Cette association peut se faire de différentes façons, mais celle qui permet d'étudier de façon quantitative l'épidermisation consiste à utiliser comme source de kératinocytes des petits explants cutanés de 1 ou $2 \mathrm{~mm}$ de diamètre. La mise en culture de l'épiderme est alors réalisée soit par l'implantation d'un explant de peau complète au centre du gel de collagène qui se contracte autour de lui, soit par collage d'un explant de peau complète ou d'épiderme seul à la surface du derme équivalent une fois la contraction stabilisée. C'est alors à partir de cet explant que l'épiderme va s'étendre progressivement à la surface du derme équivalent. L'évaluation quantitative de l'épidermisation est obtenue par la mesure de la surface épidermisée, de son contenu en $A D N$, ainsi que par la mesure de l'incorporation de thymidine. Le degré de différenciation peut également être évalué par l'étude de l'expression des kératines de haut poids moléculaire (65-67 kDa), marqueurs de la différenciation terminale de l'épiderme, par la mesure de la quantité d'ADN par unité de surface, indicateur du nombre de couches nucléées de l'épiderme, et bien évidemment par l'analyse morphologique de l'épiderme néoformé. Comme cela est montré dans la figure 3, on peut également utiliser (encarts du bas) comme matrice un gel de collagène sans fibroblastes, ou contenant des fibroblastes tués avant ou après la réorganisation. 


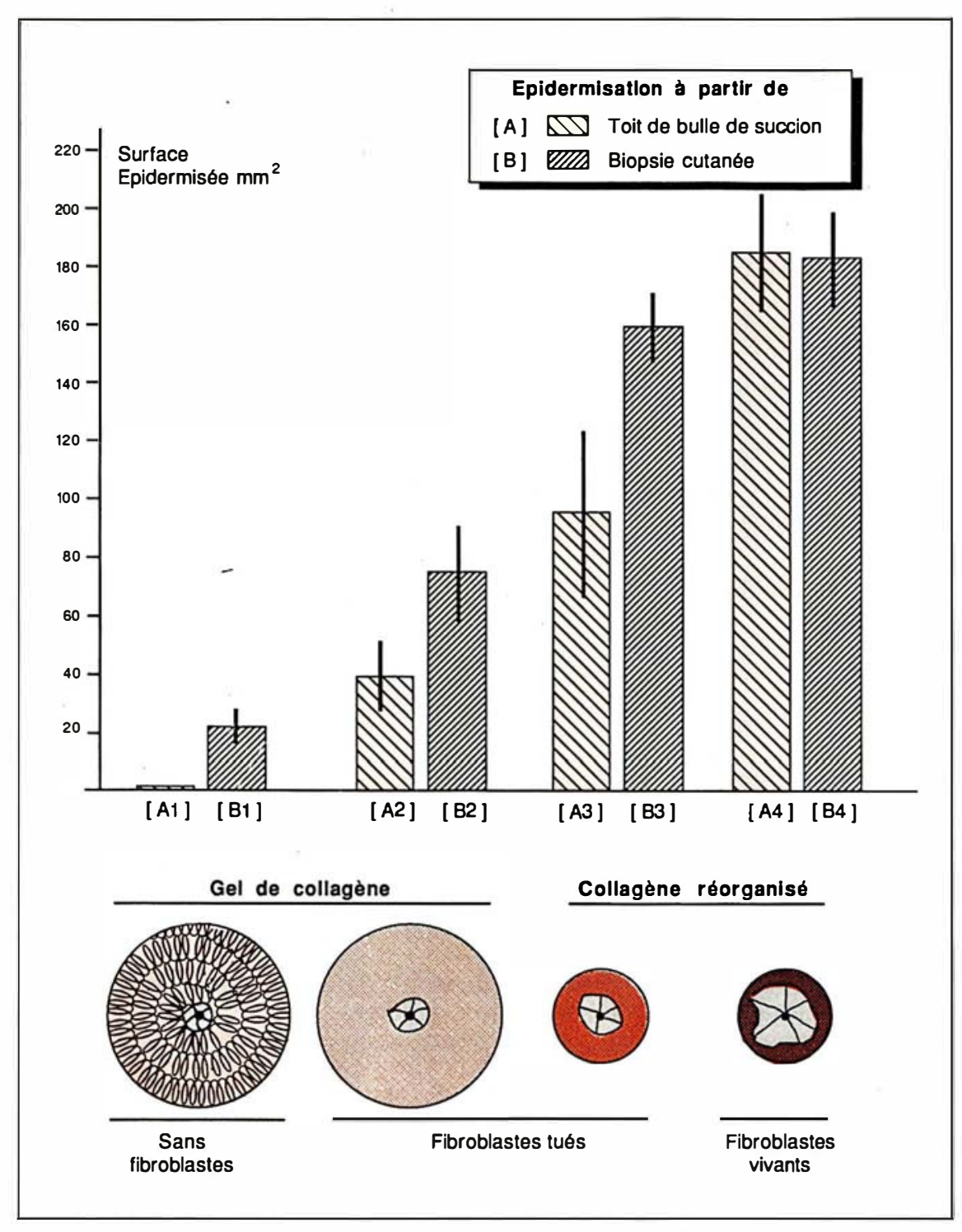

Figure 3. Influence des fibroblastes normaux sur la croissance épidermique. Un explant cutané est collé sur différents supports: soit sur un simple gel de collagène sans cellule, soit sur un gel dans lequel les fibroblastes ont été tués immédiatement après sa polymérisation, soit sur une matrice de collagène préalablement remaniée par des fibroblastes avant qu'ils ne subissent un choc osmotique, soit encore sur un derme équivalent au sens strict, dans lequel la matrice est réorganisée par des fibroblastes qui sont laissés vivants. L'épidermisation obtenue à partir de toits de bulle de succion, c'est-à-dire d'épiderme sans composant dermique montre: (1) que la texture de la matrice de collagène joue un rôle important sur l'épidermisation, la croissance épidermique étant supérieure sur une matrice réorganisée par des fibroblastes ([A2] et [A3]); (2) que la présence de fibroblastes, même tués, favorise la croissance épidermique pour une même texture de collagène ([A1] et [A2]). Cependant, c'est en présence de fibroblastes vivants que la croissance épidermique est la meilleure ([A3] et [A4]). Les résultats obtenus par l'épidermisation d'explants de peau complète, comportant derme et épiderme, vont dans le même sens ([B1], [B2] et [B3]). Cependant, les fibroblastes de cet explant interfèrent et rendent difficilement discernable le rôle des fibroblastes du derme équivalent ([B3] et [B4]). Les fibroblastes agissent donc sur la croissance de l'épiderme non seulement en remodelant la matrice de collagène qui va à son tour influencer l'épidermisation, mais également vraisemblablement en sécrétant des facteurs diffusibles qui favorisent la croissance épidermique. collagénase anormale est sécrétée en grande quantité [12] aboutissant à une destruction cutanée localisée.

L'épiderme. Le derme équivalent est également, a priori, un excellent support pour la croissance des kératinocytes (figure 2). Le premier à avoir attiré l'attention sur l'importance de facteurs d'origine fibroblastique pour la croissance des kératinocytes in vitro est H. Green travaillant lui aussi au MIT. H. Green, en 1975 a décrit avec J.Rheinwald[13] une méthode permettant de cultiver et surtout de subcultiver de grandes quantités de kératinocytes sur des lits de cellules fibroblastoïdes, les 3T3 préalablement irradiées. Dans ces cultures on voit les kératinocytes se diviser rapidement aux dépens des cellules 3T3 qui leur apportent des facteurs de croissance de nature indéterminée. Il était donc normal d'espérer pouvoir cultiver des kératinocytes sur des dermes équivalents.

Dans un premier temps ceci a été réalisé par $E$. Bell et son équipe [14] en ensemençant des kératinocytes dissociés par la trypsine sur des dermes équivalents. On voit alors se reconstituer un feuillet épidermique comportant plusieurs couches cellulaires et l'ébauche d'une couche cornée. Cette méthode a été modifiée et améliorée par notre équipe en vue d'études pharmacologiques avec des cellules humaines. La technique que nous avons mise au point consiste à implanter une petite biopsie circulaire d'épiderme ou de peau complète de 1 à $2 \mathrm{~mm}$ de diamètre au centre d'un derme équivalent [15]. A partir de cet explant, un néoépiderme se développe progressivement à la surface du derme équivalent, le couvrant progressivement, de façon circulaire et excentrique. Ce dispositif expérimental permet d'évaluer quantitativement la croissance épidermique par mesure de la surface épidermique en fonction du temps, par mesure de son contenu en ADN et par mesure de l'incorporation de la thymidine tritiée. La qualité de la différenciation 


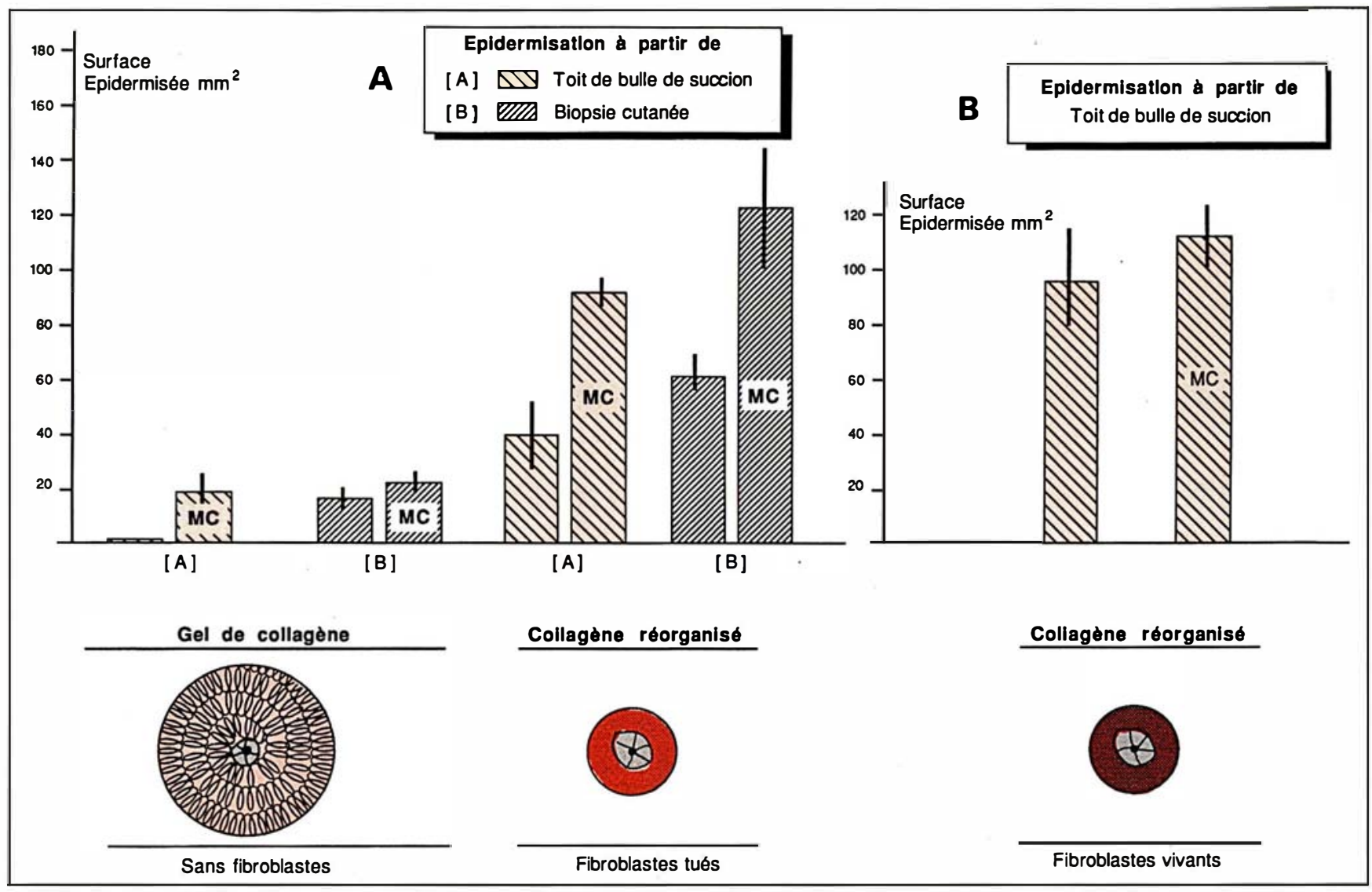

Figure 4. Influence du milieu conditionné par des fibroblastes sur la croissance épidermique : sécrétion de facteurs diffusibles par fibroblastes. A. L'épidermisation a été réalisée à partir de biopsie de peau complète (derme + épiderme: [B]) ou de toit de bulle de succion (épiderme seul: [A]), sur un simple gel de collagène, ou sur une matrice de collagène réorganisée mais dépourvue de cellules vivantes. Le traitement de ces cultures par du milieu conditionné par des fibroblastes cultivés en monocouche (MC) augmente la croissance épidermique. B. En revanche, l'addition de milieu conditionné (MC) dans des cultures où les fibroblastes sont vivants dans le derme équivalent ne provoque pas d'augmentation significative de la croissance épidermique. Les fibroblastes cultivés en monocouche, ou dans une matrice de collagène, sécrétent donc des facteurs diffusibles capables d'augmenter la croissance épidermique.

épidermique peut également être abordée soit en calculant la quantité d'ADN par $\mathrm{mm}^{2}$ ce qui donne une idée du nombre de couches de kératinocytes présentes dans le nouvel épiderme, soit en faisant une électrophorèse des kératines ce qui permet d'évaluer la présence ou non de kératines de haut poids moléculaire, marqueurs de la différenciation terminale, soit mieux encore en faisant une étude histologique de l'épiderme reconstruit, ce qui permet de comparer ce nouvel épiderme avec l'épiderme humain normal. Nous disposons donc ainsi pour la première fois d'un équivalent d'organe humain reconstruit de façon dynamique en laboratoire. Le système expérimental est qu'il peut être modulable. Il est en effet possible d'utiliser différents types de collagène pour reconstruire le derme et d'associer le collagène avec différentes macro-molécules. Il est possible de reconstruire ce derme avec des fibroblastes jeunes ou âgés, normaux ou pathologiques, traités ou non par différents médicaments ou par des rayonnements. Il est possible enfin d'associer aux fibroblastes d'autres cellules, en particulier des mastocytes (des expériences préliminaires nous ont permis de montrer que les mastocytes survivaient plus longtemps en présence de fibroblastes), des cellules endothéliales (E. Bell a pu montrer que des structures canalaires ressem- blant à des néo-capillaires se formaient alors à travers une coopération fibroblastes/cellules endothéliales) ou des plaquettes sanguines si l'on veut étudier certains des facteurs initiaux de la cicatrisation.

\section{Communications dermo-épidermiques}

Mais l'intérêt principal de ce modèle expérimental est sans doute de pouvoir étudier des interactions entre le derme et l'épiderme. Nous avons étudié ces interactions tout d'abord avec des fibroblastes normaux puis avec des fibroblastes pathologiques.

Dans une première série d'expériences, nous avons comparé la 
cinétique d'épidermisation sur 4 substrats différents : (1) un gel de collagène sans fibroblaste; (2) un gel de collagène où les fibroblastes ont été tués par choc osmotique avant contraction ; (3) un derme équivalent où les fibroblastes ont été tués par choc osmotique en fin de contraction ; (4) un derme équivalent dans lequel les fibroblastes étaient vivants (figure 3, page 105). Nous avons pu montrer ainsi que les fibroblastes contribuaient de deux façons à la croissance de l'épiderme : en remodelant la matrice de collagène, et la croissance de l'épiderme était toujours plus rapide en fin de contraction lorsque la matrice de collagène est réorganisée par les fibroblastes que sur gel simple; mais aussi en sécrétant des facteurs de croissance importants pour la croissance épidermique, et la croissance épidermique était toujours plus rapide lorsque les fibroblastes étaient vivants que lorsque les fibroblastes étaient tués par choc osmotique. Ces facteurs de croissance peuvent agir à distance. En effet, la croissance de l'épiderme est équivalente sur un derme vivant ou sur un derme dans lequel les fibroblastes ont été tués mais où le milieu de culture a été préalablement conditionné par des fibroblastes vivants (figure 4).

L'importance des interactions dermo-épidermiques est également grande en pathologie comme l'ont montré nos études sur le psoriasis [16]. Le psoriasis est une affection cutanée fréquente touchant 2 à $3 \%$ de la population mondiale et se traduisant essentiellement, cliniquement et histologiquement, par une prolifération de l'épiderme (figure 5). A cette prolifération s'associent une production de messages chimiotactiques qui provoque la migration de polynucléaires neutrophiles dans l'épiderme, et la formation de micro-abcès qui aggravent la désorganisation épidermique. Cette maladie évolue dans un contexte génétique avec transmission $\mathrm{m} / \mathrm{s} n^{\circ} 2$ vol. 4, février 88 familiale fréquente et augmentation de fréquence de certains groupes HLA. Elle est modulée dans sa gravité par des facteurs d'environnement dont les mieux connus sont les stress, les saisons, les épisodes infectieux et certains médicaments.

J.E. Fraki, en 1983 [17], alors qu'il travaillait dans l'équipe de G. Lazarus à Philadelphie, a montré que les anomalies caractéristiques du psoriasis persistaient après greffe chez la souris nude. Cela permettait donc de démontrer pour la première fois que les anomalies initiales du psoriasis se situaient bien dans la peau même si ces anomalies pouvaient être modulées par des facteurs systémiques. Restait à mieux comprendre si l'anomalie initiale se trouvait au niveau de l'épiderme ou du derme. Pour répondre à cette question, nous avons utilisé le modèle de la peau reconstruite in vitro [16], en étudiant toutes les combinaisons possibles entre des dermes réalisés avec des fibroblastes normaux, avec des fibroblastes prove- nant de peau apparemment non lésée de psoriasiques et avec des fibroblastes provenant de lésions psoriasiques, et des épidermes provenant soit de peau normale, soit de peau apparemment non lésée de psoriasiques, soit de lésions psoriasiques. Nous avons pu ainsi montrer que, certes, les kératinocytes psoriasiques provenant de lésions continuent d'hyperproliférer, même sur des dermes normaux, mais surtout que des épidermes parfaitement normaux subissent une hyperprolifération lorsqu'ils sont cultivés sur des dermes réalisés avec des fibroblastes psoriasiques. Cette hyperprolifération se manifeste de la même façon si les fibroblastes proviennent de peau lésée ou non lésée. Ainsi l'anomalie initiale du psoriasis se situe vraisemblablement au niveau des fibroblastes. Le psoriasis peut donc être considéré comme une maladie génétique du dialogue dermo-épidermique.

Il est intéressant de noter que ces résultats n'ont pu être observés qu'avec du sérum humain, ce qui

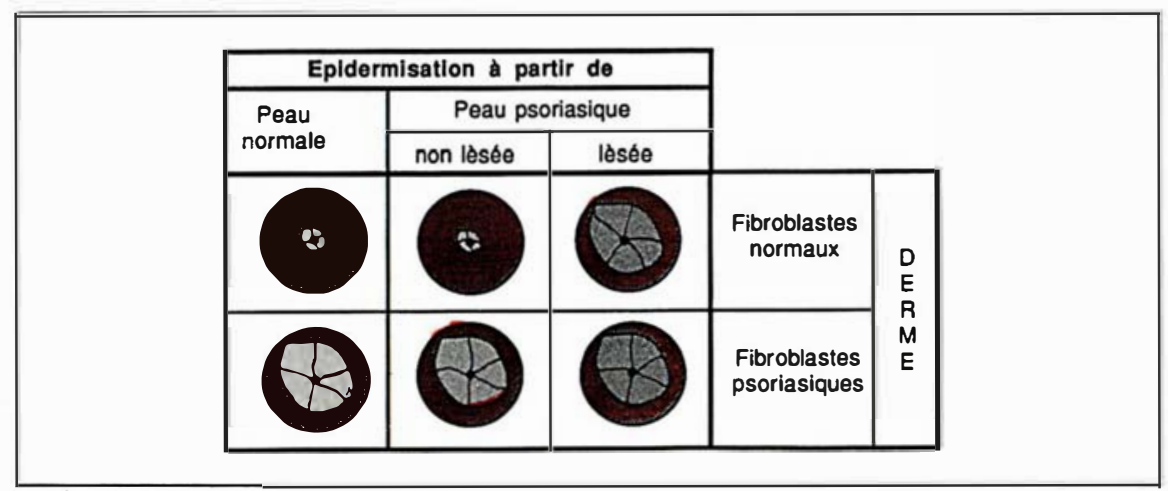

Figure 5. La peau équivalente, modèle d'étude de la physiopathologie cutanée. II est possible de profiter du caractère modulable de la peau équivalente pour étudier certaines maladies cutanées. C'est ce que nous avons fait dans le cas du psoriasis, maladie qui se caractérise en particulier par une hyperprolifération de l'épiderme. Différents dermes équivalents ont été réalisés, soit avec des fibroblastes de volontaires sains, soit avec des fibroblastes de malades atteints de psoriasis. Ces différents substrats dermiques ont alors été épidermisés par des biopsies cutanées provenant de peau normale, de peau psoriasique prélevée en zone non lésée, ou encore de lésions psoriasiques. Quelle que soit la nature des fibroblastes, l'épiderme issu de plaques de psoriasis conserve son caractère hyperprolifératif, ce qui est important puisqu'il n'existe pas de modele animal de psoriasis. Mais surtout, les fibroblastes psoriasiques, qu'ils proviennent de zones lésées ou non, sont capables d'induire l'hyperprolifération de kératinocytes normaux. Une anomalie des fibroblastes serait donc à l'origine de l'hyperprolifération caractéristique du psoriasis. Ces résultats illustrent l'importance des interactions dermo-épidermiques, en particulier pour la régulation de la croissance épidermique, et les conséquences que peut entraîner, sur le tissu épidermique, une altération d'un des composants du derme. 
souligne le fait que, si l'anomalie initiale du psoriasis est vraisemblablement située dans les fibroblastes, cette anomalie ne s'exprime que sous l'action de facteurs systémiques qui restent à définir. Quoi qu'il en soit nous disposons maintenant d'un modèle expérimental qui devrait nous permettre de mieux comprendre les anomalies moléculaires à l'origine du psoriasis et à travers elles de mieux comprendre, au niveau moléculaire également, les facteurs intervenant dans le dialogue dermoépidermique.

D'autres applications de notre modèle peuvent être envisagées. Tout récemment nous sommes parvenus à faire migrer hors de l'explant implanté au milieu du derme équivalent non seulement des kératinocytes mais également des mélanocytes, ce qui nous offre un modèle particulièrement attrayant pour étudier cette coopération cellulaire qui, à travers le dialogue mal compris kératinocytes-mélanocytes, aboutit à la pigmentation cutanée.

A travers cette recherche, la peau apparaît comme un organe modèle, véritable système modulaire biologique pouvant être reconstruit de façon simple ou complexe dans des conditions toujours plus proches de ce que l'on observe in vivo. De cette recherche devrait naître non seulement une meilleure compréhension de la physiologie et de la physiopathologie cutanée mais également une meilleure compréhension des processus de cicatrisation, de l'angiogenèse, de l'hypersensibilité immédiate et, sur un plan plus fondamental, de coopération entre cellules différenciées.

Le concept moteur de cette recherche peut être appliqué à d'autres organes $[18,19]$. C'est un concept simple: si l'on veut étudier la physiologie cellulaire en culture et obtenir des résultats extrapolables à la situation in vivo, il faut replacer les cellules in vitro dans un environnement physico-chimique le plus semblable possible à celui qui est le leur in vivo

\section{Summary}

The development of a human living skin provides an original system for studying in vitro cell biology at the tissue and organ level, in defined cells-cells and cells-matrix associations.

The skin equivalent, made with normal or pathological cells is constituted in two steps: the first one provides a dermal equivalent, the second an overlying epidermis. The dermal equivalent is by itself an original culture model. As a matter of fact, fibroblasts cultivated into a three dimensional collagen matrix express an in vivolike differentiation. Furthermore, it is possible to quantitatively evaluate cell functions that cannot be approached in other culture systems (such as fibroblasts contraction), and to modulate these functions by pharmacological agents. The association of fibroblasts and collagen, that are both involved in the promotion of the epidermal growth and differentiation, leads the dermal equivalent to be an excellent substrate for epidermalization. The use of small skin biopsies as a source of epidermalization provides a cultivated epidermis close to the in vivo one. In addition, its proliferation and its differentiation can be quantitatively evaluated. The main interest of this living skin is to be defined, at the level of the dermal and epidermal components as well as at the level of the dermal-epidermal associations.

By this way we have identified functions of normal fibroblasts that promote epidermalization, and demonstrated that psoriatic fibroblasts are able to induce the hyperproliferation of normal keratinocytes. Thus this dermalepidermal interactions model is an important tool for human skin physiology, physiopathology and pharmacology. 